\title{
REPRESENTATION FORMULAS FOR ENTIRE FUNCTIONS OF EXPONENTIAL TYPE AND GENERALIZED BERNOULLI POLYNOMIALS
}

\author{
C. FRAPPIER \\ (Received 21 March 1997; revised 28 November 1997)
}

Communicated by P. C. Fenton

\begin{abstract}
We introduce a sequence of polynomials which are extensions of the classic Bernoulli polynomials. This generalization is obtained by using the Bessel functions of the first kind. We use these polynomials to evaluate explicitly a general class of series containing an entire function of exponential type.
\end{abstract}

1991 Mathematics subject classification (Amer. Math. Soc.): primary 30D10, 33C10, 12 E10.

\section{Introduction}

We denote by $E_{\tau}\left(\delta_{0}\right)$ the class of all entire functions of exponential type $\tau$ ( $\tau>0$ ) such that $f(x)=O\left(|x|^{-\delta}\right)$, as $x \rightarrow \pm \infty$, for some $\delta>\delta_{0}$. The indicator function of an entire function of exponential type is defined as

$$
h_{f}(\theta):=\limsup _{r \rightarrow \infty} \frac{\ln \left|f\left(r e^{i \theta}\right)\right|}{r}, \quad-\pi \leq \theta \leq \pi .
$$

The following results are proved in [4].

THEOREM A. Given an integer $n \geq 2$, let $f \in E_{\tau}(1-n)$ be such that $h_{f}(\pi / 2) \leq 0$. We have, for $\sigma \geq \tau$ and $0 \leq x \leq 1-\tau / \sigma$,

$$
-n !\left(\frac{\sigma}{2 \pi}\right)^{n} \sum_{\substack{k=\infty \\
k \neq 0}}^{\infty} \frac{e^{2 k \pi i x}}{k^{n}} f\left(\frac{2 k \pi}{\sigma}\right)=\sum_{j=0}^{n}\left(\begin{array}{l}
n \\
j
\end{array}\right) B_{j}(x)(i \sigma)^{j} f^{(n-j)}(0) .
$$

(C) 1998 Australian Mathematical Society 0263-6115/98 $\$$ A2.00+0.00 
Here $B_{j}(x)$ is the $j$ th Bernoulli polynomial defined by the generating function

$$
\frac{z e^{x z}}{e^{z}-1}=\sum_{n=0}^{\infty} B_{n}(x) \frac{z^{n}}{n !}, \quad|z|<2 \pi .
$$

It is well known $[1, \mathrm{p} .267]$ that

$$
B_{n}(x)=\frac{-n !}{(2 \pi i)^{n}} \sum_{\substack{k=-\infty \\ k \neq 0}}^{\infty} \frac{e^{2 k \pi i x}}{k^{n}}, \quad 0 \leq x \leq 1,
$$

which is a very special case of (2).

THEOREM B. Given an integer $n \geq 2$, let $f \in E_{\tau}(1-n)$. We have, for $\sigma \geq 2 \tau$ and $0 \leq x \leq 1-2 \tau / \sigma$,

(5)

$$
-n !\left(\frac{\sigma}{2 \pi}\right)^{n} \sum_{\substack{k=-\infty \\
k \neq 0}}^{\infty} \frac{e^{2 k \pi i(x+\tau / \sigma)}}{k^{n}} f\left(\frac{2 k \pi}{\sigma}\right)=\sum_{j=0}^{n}\left(\begin{array}{l}
n \\
j
\end{array}\right) B_{j}\left(x+\frac{\tau}{\sigma}\right)(i \sigma)^{j} f^{(n-j)}(0) .
$$

The special cases $\sigma=\tau$ of (2) and $\sigma=2 \tau$ of (5) have been investigated previously in [3]. The numbers $B_{j}(0)=B_{j}$ are the Bernoulli numbers.

In this paper we generalize (2) and (5). In order to do that, we construct a sequence of polynomials which are direct extensions of Bernoulli (and Euler) polynomials. These generalized Bernoulli polynomials turn out to have some properties similar to the classical ones.

\section{Generalized Bernoulli polynomials}

Let $g_{\alpha}(z):=2^{\alpha} \Gamma(\alpha+1) J_{\alpha}(z) / z^{\alpha}$, where $J_{\alpha}(z)=\sum_{k=0}^{\infty}(-1)^{k} z^{2 k+\alpha} /\left(2^{2 k+\alpha} k ! \Gamma(\alpha+\right.$ $k+1)$ ) is the Bessel function of the first kind of order $\alpha$. The function $J_{\alpha}(z) / z^{\alpha}$ is an even entire function of exponential type one. We assume that $\alpha$ is not a negative integer. The zeros $j_{k}=j_{k}(\alpha)$ of $J_{\alpha}(z) / z^{\alpha}$ may then be ordered in such a way that $j_{-k}=-j_{k}$ and $0<\left|j_{1}\right| \leq\left|j_{2}\right| \leq \ldots$

We define a sequence of polynomials $B_{n, \alpha}(x)$ by the generating function

$$
\frac{e^{(x-1 / 2) z}}{g_{\alpha}(i z / 2)}=\sum_{n=0}^{\infty} B_{n, \alpha}(x) \frac{z^{n}}{n !}, \quad|z|<2\left|j_{1}\right| .
$$

It is clear from (6) that $B_{n, \alpha}(x)$ is a rational fraction in $\alpha$. We call the polynomials $B_{n, \alpha}(x)$ the $\alpha$-Bernoulli polynomials and $B_{n, \alpha}(0)=: B_{n, \alpha}$ the $\alpha$-Bernoulli numbers. 
Equation (6) reduces to (3) for $\alpha=1 / 2$ since $J_{1 / 2}(z)=\sqrt{2 / \pi z} \sin (z)$. In the case $\alpha=-1 / 2$ we have $J_{-1 / 2}(z)=\sqrt{2 / \pi z} \cos (z)$ and (6) becomes

$$
\frac{2 e^{x z}}{e^{z}+1}=\sum_{n=0}^{\infty} B_{n,-1 / 2}(x) \frac{z^{n}}{n !}, \quad|z|<\pi
$$

So, $B_{n,-1 / 2}(x)=E_{n}(x)$ is the Euler polynomial of degree $n$. For $\alpha=3 / 2$, Equation (6) reduces to

$$
\frac{1}{6} \cdot \frac{z^{3} e^{x z}}{(z-2) e^{z}+(z+2)}=\sum_{n=0}^{\infty} B_{n, 3 / 2}(x) \frac{z^{n}}{n !} .
$$

For an arbitrary $\alpha$ (not a negative integer) we have $B_{0, \alpha}(x)=1, B_{1, \alpha}(x)=x-1 / 2$, $B_{2, \alpha}(x)=(x-1 / 2)^{2}-1 / 8(\alpha+1), B_{3, \alpha}(x)=(x-1 / 2)^{3}-3(x-1 / 2) / 8(\alpha+1)$, so that $B_{0, \alpha}=1, B_{1, \alpha}=-1 / 2, B_{2, \alpha}=(2 \alpha+1) / 8(\alpha+1), B_{3, \alpha}=(1-2 \alpha) / 16(\alpha+1)$.

REMARK 1. It follows from the asymptotic relation [7, p. 225]

$$
\frac{J_{\alpha}(z)}{z^{\alpha}} \sim \frac{1}{\sqrt{2 \pi \alpha}} \frac{e^{\alpha}}{(2 \alpha)^{\alpha}}, \quad \alpha \rightarrow \infty \quad(\text { real }),
$$

that

$$
\lim _{\alpha \rightarrow \infty} B_{n, \alpha}(x)=(x-1 / 2)^{n} .
$$

The following result contains some basic properties of the $\alpha$-Bernoulli polynomials and numbers. We omit the details of the proof since it follows exactly the same line as in the case $\alpha=1 / 2$ (see [1] or any of the numerous books on the subject).

PROPOSITION. For any complex number $\alpha$ (not a negative integer), we have

$$
B_{n, \alpha}^{\prime}(x)=n B_{n-1 . \alpha}(x), \quad n=1,2,3, \ldots
$$

$$
B_{n, \alpha}(1-x)=(-1)^{n} B_{n, \alpha}(x), \quad n=0,1,2, \ldots
$$

(In particular, $B_{n, \alpha}(1)=(-1)^{n} B_{n, \alpha}$ and $\left.B_{2 m+1, \alpha}(1 / 2)=0, m=0,1,2, \ldots\right)$

$$
\begin{aligned}
& B_{n, \alpha}(x+y)=\sum_{j=0}^{n}\left(\begin{array}{l}
n \\
j
\end{array}\right) B_{j, \alpha}(y) x^{n-j}, \quad n=0,1,2, \ldots \\
& \text { (In particular, } \left.B_{n, \alpha}(x)=\sum_{j=0}^{n}\left(\begin{array}{l}
n \\
j
\end{array}\right) B_{j, \alpha} x^{n-j} .\right)
\end{aligned}
$$


REMARK 2. The third part of the proposition contains, as a limiting case, the binomial formula. Indeed, if we let $\alpha \rightarrow \infty$ in (12) then we obtain, in view of (9), $(x+y-1 / 2)^{n}=\sum_{j=0}^{n}\left(\begin{array}{c}n \\ j\end{array}\right)(y-1 / 2)^{j} x^{n-j}$.

Our first theorem is the extended version of (4).

THEOREM 1. Let $n$ be a non-negative integer and $\alpha$ a complex number such that $\operatorname{Re}(\alpha)<n-1 / 2, \alpha \neq-1,-2, \ldots$ We have, for $0 \leq x \leq 1$,

$$
B_{n, \alpha}(x)=\frac{-n !}{2^{\alpha} \Gamma(\alpha+1)(2 i)^{n}} \sum_{\substack{k=-\infty \\ k \neq 0}}^{\infty} \frac{e^{(2 x-1) i j_{k}}}{j_{k}^{n-\alpha+1} J_{\alpha}^{\prime}\left(j_{k}\right)} .
$$

COROLLARY 1.1. Let $m$ be a non-negative integer and $\alpha$ a complex number such that $\operatorname{Re}(\alpha)<2 m-1 / 2, \alpha \neq-1,-2, \ldots$ We have, for $0 \leq x \leq 1$,

$$
B_{2 m, \alpha}(x)=\frac{2(-1)^{m+1}(2 m) !}{2^{\alpha} \Gamma(\alpha+1) 2^{2 m}} \sum_{k=1}^{\infty} \frac{\cos \left((2 x-1) j_{k}\right)}{j_{k}^{2 m-\alpha+1} J_{\alpha}^{\prime}\left(j_{k}\right)} .
$$

COROLLARY 1.2. Let $m$ be a non-negative integer and $\alpha$ a complex number such that $\operatorname{Re}(\alpha)<2 m+1 / 2, \alpha \neq-1,-2, \ldots$ We have, for $0 \leq x \leq 1$,

$$
B_{2 m+1, \alpha}(x)=\frac{(-1)^{m+1}(2 m+1) !}{2^{\alpha} \Gamma(\alpha+1) 2^{2 m}} \sum_{k=1}^{\infty} \frac{\sin \left((2 x-1) j_{k}\right)}{j_{k}^{2 m-\alpha+2} J_{\alpha}^{\prime}\left(j_{k}\right)} .
$$

Equations (14) and (15) follow from (13) and the relation [7, p. 75, Formula (1)] $J_{\alpha}^{\prime}\left(z e^{l \pi i}\right)=e^{(\alpha-1) l \pi i} J_{\alpha}^{\prime}(z)$, for integral $l$. Equation (14) may also be deduced from (15) and (10).

PROOF OF THEOREM 1. Given $g \in E_{\tau}(\operatorname{Re}(\alpha)+1 / 2)$ we have [5], for all $z$,

$$
g(z)=\frac{J_{\alpha}(\tau z)}{(\tau z)^{\alpha}} \sum_{\substack{k=-\infty \\ k \neq 0}}^{\infty} \frac{j_{k}^{\alpha} g\left(j_{k} / \tau\right)}{\left(\tau z-j_{k}\right) J_{\alpha}^{\prime}\left(j_{k}\right)} .
$$

If $f \in E_{2 \tau}(\operatorname{Re}(\alpha)+1 / 2)$ and $h_{f}(\pi / 2) \leq 0$ then the function $g(z):=e^{-i \tau z} f(z)$ is an element of $E_{\tau}(\operatorname{Re}(\alpha)+1 / 2)$; this result is a consequence of [2, Theorem 6.2.4]. Applying (16) we obtain, for all $z$,

$$
f(z)=e^{i \tau z} \frac{J_{\alpha}(\tau z)}{(\tau z)^{\alpha}} \sum_{\substack{k=\infty \\ k \neq 0}}^{\infty} \frac{j_{k}^{\alpha} f\left(j_{k} / \tau\right)}{\left(\tau z-j_{k}\right) e^{i j_{k}} J_{\alpha}^{\prime}\left(j_{k}\right)} .
$$

In (17), let $\tau=1$ and $f(z)=e^{2 i x z}, 0 \leq x \leq 1$. This gives us

$$
e^{(2 x-1) i z}=\frac{J_{\alpha}(z)}{z^{\alpha}} \sum_{\substack{k=-\infty \\ k \neq 0}}^{\infty} \frac{j_{k}^{\alpha} e^{(2 x-1) i j_{k}}}{\left(z-j_{k}\right) J_{\alpha}^{\prime}\left(j_{k}\right)},
$$


for $\operatorname{Re}(\alpha)<-1 / 2$, whence

$$
\frac{1}{n !}\left[\left(e^{(2 x-1) i z} \frac{z^{\alpha}}{J_{\alpha}(z)}\right)^{(n)}\right]_{z=0}=-\sum_{\substack{k=-\infty \\ k \neq 0}}^{\infty} \frac{e^{(2 x-1) i j_{k}}}{j_{k}^{n-\alpha+1} J_{\alpha}^{\prime}\left(j_{k}\right)}
$$

for $\operatorname{Re}(\alpha)<n-1 / 2$.

On the other hand, it follows directly from (6) that

$$
B_{n, \alpha}(x)=\left[\left(\frac{e^{(2 x-1) z / 2}(-i z / 2)^{\alpha}}{2^{\alpha} \Gamma(\alpha+1) J_{\alpha}(-i z / 2)}\right)^{(n)}\right]_{z=0}=\left(-\frac{i}{2}\right)^{n}\left[\left(\frac{e^{(2 x-1) i z} z^{\alpha}}{2^{\alpha} \Gamma(\alpha+1) J_{\alpha}(z)}\right)^{(n)}\right]_{z=0},
$$

which gives the result by comparison with (19).

\section{Representation formulas}

The results given in this section contain Theorem A and B as the special case $\alpha=1 / 2$.

THEOREM 2. Let $n$ be a non-negative integer and $\alpha$ a complex number such that $\operatorname{Re}(\alpha)<n-1 / 2, \alpha \neq-1,-2, \ldots$ For all $f \in E_{\tau}(\operatorname{Re}(\alpha)+1 / 2-n)$ such that $h_{f}(\pi / 2) \leq 0$, we have, for $\sigma \geq \tau$ and $0 \leq x \leq 1-\tau / \sigma$,

$$
\frac{-n !(\sigma / 2)^{n}}{2^{\alpha} \Gamma(\alpha+1)} \sum_{\substack{k=-\infty \\
k \neq 0}}^{\infty} \frac{e^{(2 x-1) i j_{k}} f\left(2 j_{k} / \sigma\right)}{j_{k}^{n-\alpha+1} J_{\alpha}^{\prime}\left(j_{k}\right)}=\sum_{j=0}^{n}\left(\begin{array}{l}
n \\
j
\end{array}\right) B_{j, \alpha}(x)(i \sigma)^{j} f^{(n-j)}(0)
$$

PROOF. We first prove the result for $\sigma=\tau$. In view of the theorem of Paley and Wiener [2, p. 103], the entire function $f$ is of exponential type $\tau$ and belongs to $L^{2}(-\infty, \infty)$ if and only if

$$
f(z)=\int_{-\tau}^{\tau} e^{i z t} \phi(t) d t
$$

where $\phi \in L^{2}(-\tau, \tau)$. If $h_{f}(\pi / 2) \leq 0$ then the representation (22) takes the form (see $[6$, p. 387])

$$
f(z)=\int_{0}^{\tau} e^{i z t} \phi(t) d t
$$


where $\phi \in L^{2}(0, \tau)$. In that case, we have

$$
\begin{aligned}
\sum_{\substack{k=-\infty \\
k \neq 0}}^{\infty} \frac{f\left(2 j_{k} / \tau\right)}{j_{k}^{n-\alpha+1} e^{i j_{k}} J_{\alpha}^{\prime}\left(j_{k}\right)} & =\sum_{\substack{k=-\infty \\
k \neq 0}}^{\infty} \frac{1}{j_{k}^{n-\alpha+1} e^{i j_{k}} J_{\alpha}^{\prime}\left(j_{k}\right)} \int_{0}^{\tau} e^{2 i j_{k} t / \tau} \phi(t) d t \\
& =-2^{\alpha} \Gamma(\alpha+1) \frac{(2 i)^{n}}{n !} \int_{0}^{\tau} B_{n, \alpha}(t / \tau) \phi(t) d t,
\end{aligned}
$$

by Theorem 1 . The interchange in the order of summation and integration is easily justifiable for $\operatorname{Re}(\alpha)<n-1 / 2$. Using (12) with $x=t / \tau, y=0$, we may thus write

$$
\begin{aligned}
\sum_{\substack{k=-\infty \\
k \neq 0}}^{\infty} \frac{f\left(2 j_{k} / \tau\right)}{j_{k}^{n-\alpha+1} e^{i j_{k}} J_{\alpha}^{\prime}\left(j_{k}\right)} & =-2^{\alpha} \Gamma(\alpha+1) \frac{(2 i)^{n}}{n !} \int_{0}^{\tau} \sum_{j=0}^{n}\left(\begin{array}{c}
n \\
j
\end{array}\right) B_{j, \alpha}(t / \tau)^{n-j} \phi(t) d t \\
& =-2^{\alpha} \Gamma(\alpha+1) \frac{(2 i)^{n}}{n !} \sum_{j=0}^{n}\left(\begin{array}{c}
n \\
j
\end{array}\right) B_{j, \alpha}(i \tau)^{j-n} f^{(n-j)}(0)
\end{aligned}
$$

by (23). Hence,

$$
\frac{-n !(\tau / 2)^{n}}{2^{\alpha} \Gamma(\alpha+1)} \sum_{\substack{k=\infty \\
k \neq 0}}^{\infty} \frac{f\left(2 j_{k} / \tau\right)}{j_{k}^{n-\alpha+1} e^{i j_{k}} J_{\alpha}^{\prime}\left(j_{k}\right)}=\sum_{j=0}^{n}\left(\begin{array}{c}
n \\
j
\end{array}\right) B_{j . \alpha}(i \tau)^{j} f^{(n-j)}(0) .
$$

Thus, Equation (21) is proved for $\sigma=\tau$ whenever $f$ belongs to $L^{2}(-\infty, \infty)$. If $f$ does not belong to $L^{2}(-\infty, \infty)$ then we need only apply (24) to a function of the form $f_{\varepsilon}(z):=\left(\left(e^{i \varepsilon z}-1\right) / i \varepsilon z\right)^{N} f(z), \varepsilon>0$, for some positive integer $N$, and let $\varepsilon \rightarrow 0$. The passage to the limit is easily justifiable.

We now prove the general result. We have, using (12) with $y=0$,

$$
\begin{aligned}
\sum_{j=0}^{n}\left(\begin{array}{l}
n \\
j
\end{array}\right) B_{j, \alpha}(x)(i \sigma)^{j} f^{(n-j)}(0) \\
\quad=\sum_{j=0}^{n} \sum_{k=0}^{j}\left(\begin{array}{l}
n \\
j
\end{array}\right)\left(\begin{array}{l}
j \\
k
\end{array}\right) B_{k, \alpha} x^{j-k}(i \sigma)^{j} f^{(n-j)}(0) \\
=\sum_{j=0}^{n} \sum_{k=0}^{j}\left(\begin{array}{c}
n \\
k+j
\end{array}\right)\left(\begin{array}{c}
k+j \\
j
\end{array}\right) B_{j, \alpha} x^{k}(i \sigma)^{k+j} f^{(n-k-j)}(0) \\
=\sum_{j=0}^{n}\left(\begin{array}{c}
n \\
j
\end{array}\right) B_{j, \alpha}(i \sigma)^{j}\left(e^{i \sigma x w} f(w)\right)^{(n-j)}(w=0),
\end{aligned}
$$

by Leibniz's formula. The relation $\left(\begin{array}{c}n \\ k+j\end{array}\right)\left(\begin{array}{c}k+j \\ j\end{array}\right)=\left(\begin{array}{c}n \\ j\end{array}\right)\left(\begin{array}{c}n-j \\ k\end{array}\right)$ has also been used. We apply (24) where $f(z)$ is replaced by $e^{i \sigma x z} f(z), x \geq 0$, which is of exponential type 
$\leq \sigma$ if $x \leq 1-\tau / \sigma$. We obtain

$$
\sum_{j=0}^{n}\left(\begin{array}{l}
n \\
j
\end{array}\right) B_{j, \alpha}(x)(i \sigma)^{j} f^{(n-j)}(0)=\frac{-n !(\sigma / 2)^{n}}{2^{\alpha} \Gamma(\alpha+1)} \sum_{\substack{k=-\infty \\
k \neq 0}}^{\infty} \frac{e^{2 i x j_{k}} f\left(2 j_{k} / \sigma\right)}{j_{k}^{n-\alpha+1} e^{i j_{k}} J_{\alpha}^{\prime}\left(j_{k}\right)}
$$

which is the general result.

As a consequence of Theorem 2, we are now able to prove

THEOREM 3. Let $n$ be a non-negative integer and $\alpha$ a complex number such that $\operatorname{Re}(\alpha)<n-1 / 2, \alpha \neq-1,-2, \ldots$ For all $f \in E_{\tau}(\operatorname{Re}(\alpha)+1 / 2-n)$, we have, for $\sigma \geq 2 \tau$ and $0 \leq x \leq 1-2 \tau / \sigma$,

$$
\frac{-n !(\sigma / 2)^{n}}{2^{\alpha} \Gamma(\alpha+1)} \sum_{\substack{k=-\infty \\
k \neq 0}}^{\infty} \frac{e^{(2(x+\tau / \sigma)-1) i j_{k}} f\left(2 j_{k} / \sigma\right)}{j_{k}^{n-\alpha+1} J_{\alpha}^{\prime}\left(j_{k}\right)}=\sum_{j=0}^{n}\left(\begin{array}{c}
n \\
j
\end{array}\right) B_{j, \alpha}(x+\tau / \sigma)(i \sigma)^{j} f^{(n-j)}(0)
$$

ProOF. We apply Theorem 2, where $\tau$ is replaced by $2 \tau$, to the function $g(z):=$ $e^{i \tau z} f(z)$; we have $h_{g}(\pi / 2) \leq 0$ since [2, Theorem 5.4.1] $h_{f}(\theta) \leq \tau,-\pi \leq \theta \leq \pi$. We obtain, using Leibniz's formula,

$$
\begin{aligned}
& \frac{-n !(\sigma / 2)^{n}}{2^{\alpha} \Gamma(\alpha+1)} \sum_{\substack{k=-\infty \\
k \neq 0}}^{\infty} \frac{e^{(2(x+\tau / \sigma)-1) i j_{k}} f\left(2 j_{k} / \sigma\right)}{j_{k}^{n-\alpha+1} J_{\alpha}^{\prime}\left(j_{k}\right)} \\
& =\sum_{j=0}^{n} \sum_{k=0}^{n-j}\left(\begin{array}{l}
n \\
j
\end{array}\right)\left(\begin{array}{c}
n-j \\
k
\end{array}\right) B_{j, \alpha}(x)(i \sigma)^{j}(i \tau)^{n-j-k} f^{(k)}(0) \\
& =\sum_{k=0}^{n} \sum_{j=0}^{n-k}\left(\begin{array}{l}
n \\
j
\end{array}\right)\left(\begin{array}{c}
n-j \\
k
\end{array}\right) B_{j, \alpha}(x)(i \sigma)^{j}(i \tau)^{n-j-k} f^{(k)}(0) \\
& =\sum_{k=0}^{n} \sum_{j=0}^{n-k}\left(\begin{array}{l}
n \\
k
\end{array}\right)\left(\begin{array}{c}
n-k \\
j
\end{array}\right) B_{j, \alpha}(x)(i \sigma)^{j}(i \tau)^{n-j-k} f^{(k)}(0) \\
& =\sum_{k=0}^{n}\left(\begin{array}{l}
n \\
k
\end{array}\right) B_{n-k, \alpha}(x+\tau / \sigma)(i \sigma)^{n-k} f^{(k)}(0),
\end{aligned}
$$

where the last step uses the addition formula (12). The result follows by replacing $k$ by $(n-k)$.

REMARK 3. Theorem 1 is a special case of both Theorems 2 and 3 where $\sigma \rightarrow \infty$ or $f(z) \equiv 1$. 


\section{Other remarks and examples}

4.1. We take, in Theorem $3, \sigma=2 \tau$ and $f(z)=J_{\alpha}(\tau z) /(\tau z)^{\alpha}$. The resulting formula reduces to a trivial identity if $n$ is odd since $B_{2 j+1, \alpha}(1 / 2)=0, j=0,1,2, \ldots$ However, if $n=2 m$ is even then we are led to the recurrence relation

$$
\sum_{k=0}^{m} \frac{2^{4 k} B_{2 k, \alpha}(1 / 2)}{(2 k) !(m-k) ! \Gamma(\alpha+m-k+1)}=0, \quad m=1,2, \ldots,
$$

which is useful to compute $B_{2 m, \alpha}(1 / 2)$. Note that (27), being obtained as a consequence of Theorem 3, is first valid only for $\operatorname{Re}(\alpha)<2 m-1 / 2$. This relation is in fact valid for all $\alpha \neq-1,-2, \ldots$ since its left-hand member is a rational fraction in $\alpha$. As a consequence of (27) we obtain $B_{2, \alpha}(1 / 2)=-1 / 8(\alpha+1), B_{4, \alpha}(1 / 2)=$ $3(\alpha+3) / 64(\alpha+1)^{2}(\alpha+2), B_{6, \alpha}(1 / 2)=-15\left(\alpha^{2}+8 \alpha+19\right) / 512(\alpha+1)^{3}(\alpha+2)(\alpha+3)$, and so on. The relation $B_{n, \alpha}(x)=\sum_{j=0}^{n}\left(\begin{array}{c}n \\ j\end{array}\right) B_{j, \alpha}(1 / 2)(x-1 / 2)^{n-j}$ may then be used to compute the $\alpha$-Bernoulli polynomials explicitly.

4.2. Assume that $\alpha>-1$ is real. The zeros of $J_{\alpha}(z) / z^{\alpha}$ are then real and simple [7, p. 479]. It follows from Theorem 1 that, for $0 \leq x \leq 1, x \neq(2 \ell+n+1) \pi / 4 j_{1}+1 / 2$, for integral $\ell$,

$$
B_{n, \alpha}(x) \sim \frac{-n !\left(e^{(2 x-1) i j_{1}}+(-1)^{n} e^{-(2 x-1) i j_{1}}\right)}{2^{\alpha} \Gamma(\alpha+1)(2 i)^{n} j_{1}^{n-\alpha+1} J_{\alpha}^{\prime}\left(j_{1}\right)},
$$

as $n \rightarrow \infty$. For $\alpha=1 / 2\left(j_{1}=\pi\right)$ the asymptotic relation (28) gives

$$
B_{n}(x) \sim \frac{-n !}{(2 \pi i)^{n}}\left(e^{2 \pi i x}+(1)^{n} e^{-2 \pi i x}\right), \quad n \rightarrow \infty,
$$

which makes sense for all $0 \leq x \leq 1$ except for $x=1 / 4,3 / 4$ if $n$ is even. The relation (29) reduces itself to the well-known [1, p. 267] asymptotic relation

$$
B_{2 m} \sim \frac{2(-1)^{m+1}(2 m) !}{(2 \pi)^{2 m}}, \quad m \rightarrow \infty,
$$

in the particular case $x=0$.

4.3. Let $\alpha=3 / 2$ and $x=0$ in (13). We obtain, for $n \geq 3$,

$$
B_{n, 3 / 2}=\frac{-n !}{3(2 i)^{n}} \sum_{\substack{k=-\infty \\ k \neq 0}}^{\infty} \frac{\left(1-i j_{k}\right)}{j_{k}^{n}}
$$


where $\tan \left(j_{k}\right)=j_{k}$ since $\left[7\right.$, p. 54, Formula (3)] $J_{3 / 2}(z)=\sqrt{2 / \pi z}(\sin (z) / z-\cos (z))$. It follows that

$$
B_{n, 3 / 2}=\frac{-n !}{3(2 i)^{n}} \sum_{\substack{k=-\infty \\ k \neq 0}}^{\infty} \frac{1}{j_{k}^{n}}
$$

if $n$ is even, while

$$
B_{n, 3 / 2}=\frac{i n !}{3(2 i)^{n}} \sum_{\substack{k=-\infty \\ k \neq 0}}^{\infty} \frac{1}{j_{k}^{n-1}}
$$

if $n$ is odd. If $n$ is even then $(n+1)$ is odd and the summation in (33) is the same as in (32). Thus, if we replace $n$ by $(n+1)$ in (33) and compare with (32), we obtain

$$
B_{n+1,3 / 2}=\frac{-(n+1)}{2} B_{n, 3 / 2}, \quad n \geq 4, \quad \text { even. }
$$

Also, since $J_{5 / 2}(z)=\sqrt{2 / \pi z}\left(\left(3 / z^{2}-1\right) \sin (z)-3 \cos (z) / z\right)$ by Formula $(2)$ of [7, p. 53], we have

$$
B_{n .5 / 2}=\frac{-n !}{15(2 i)^{n}} \sum_{\substack{k=-\infty \\ k \neq 0}}^{\infty} \frac{\left(3-j_{k}^{2}\right)-3 i j_{k}}{j_{k}^{n}}
$$

where $\tan \left(j_{k}\right)=3 j_{k} /\left(3-j_{k}^{2}\right)$, and we deduce similarly that

$$
12 B_{n+1,5 / 2}=-6(n+1) B_{n, 5 / 2}-(n+1) n B_{n-1,5 / 2} \text {, }
$$

for all even integers $n \geq 6$.

\section{Acknowledgement}

This research was supported by the Natural Sciences and Engineering Research Council of Canada, Grant No. OGP0009331.

\section{References}

[1] T. M. Apostol, Introduction to analytic number theory (Springer, New York, 1976).

[2] R. P. Boas, Entire functions (Academic Press, New York, 1954).

[3] C. Frappier, 'Some representation formulae for entire functions of exponential type', Bull. Austral. Math. Soc. 37 (1988), 17-26. 
[4] - 'Representation formulas for integrable and entire functions of exponential type II', Canad. J. Math. 43 (1991), 34-47.

[5] H. P. Kramer, 'A generalized sampling theorem', J. Math. Phys. 63 (1957), 68-72.

[6] B. Ja. Levin, Distribution of zeros of entire functions, Transl. Math. Monographs 5 (American Math. Soc., Providence, 1964).

[7] G. N. Watson, A treatise on the theory of Bessel functions, 2nd edition (Cambridge University Press, Cambridge, 1944).

Département de Mathématiques et de Génie industriel École Polytechnique de Montréal

Campus de l'Université de Montréal

C.P. 6079, Succursale Centre-Ville

Montréal (Québec)

Canada $\mathrm{H} 3 \mathrm{C}$ 3A7

e-mail address: clement.frappier@courrier.polymtl.ca 